Pacific

Journal of

Mathematics

CATEGORY O FOR THE VIRASORO ALGEBRA: COHOMOLOGY AND KOSZULITY

Brian D. Boe, Daniel K. NAKano And Emilie Wiesner 


\title{
CATEGORY $O$ FOR THE VIRASORO ALGEBRA: COHOMOLOGY AND KOSZULITY
}

\author{
Brian D. BoE, DANiEl K. NAKAno AND EMilie Wiesner
}

\begin{abstract}
We investigate blocks of the Category 0 for the Virasoro algebra over $\mathbb{C}$. We demonstrate that the blocks have Kazhdan-Lusztig theories and that the truncated blocks give rise to interesting Koszul algebras. The simple modules have BGG resolutions, and from this we compute the extensions between Verma modules and simple modules, and between pairs of simple modules.
\end{abstract}

\section{Introduction}

Bernšteĭn, Gel'fand, and Gel'fand [1976] initiated the study of Category 0 for complex semisimple Lie algebras. Since the introduction of Category $\mathbb{O}$, much progress has been made in studying the structure of blocks for this category and its variants. One of the major results in this area was the formulation and proof of the Kazhdan-Lusztig (KL) conjectures [Kazhdan and Lusztig 1979; Beřlinson and Bernstein 1981; Brylinski and Kashiwara 1981], which provide a recursive formula for the characters of simple modules in Category $\mathbf{O}$. These conjectures have been equivalently formulated in terms of Ext-vanishing conditions between simple modules and Verma modules. For semisimple algebraic groups over fields of positive characteristic $p>0$, an analogous conjecture has been provided by Lusztig as long as $p$ is at least as large as the Coxeter number of the underlying root system. The characteristic $p$ Lusztig Conjecture still remains open.

In an attempt to better understand both the original Kazhdan-Lusztig Conjecture and the Lusztig Conjecture, Cline, Parshall, and Scott [1988; 1993; 1997] developed an axiomatic treatment of highest weight categories with the added structures involving "Kazhdan-Lustzig theories" and Koszulity. Irving [1990; 1992] has partially developed some theories along these lines. Berlinson, Ginzburg, and Soergel [1996] proved that the principal block of Category 0 is Koszul using perverse sheaves and established Koszul duality between various blocks of Category $\mathbb{O}$,

MSC2000: 17B68, 17B10, 17B56, 17B65.

Keywords: Virasoro algebra, Lie algebra cohomology, extensions.

Boe's research was partially supported by NSA grant H98230-04-1-0103. Nakano's research was partially supported by NSF grant DMS-0400548. 
which provides an alternative proof of the KL Conjecture. The work of Cline, Parshall, and Scott is important because it isolates the key homological criteria for verifying the existence of such properties.

The Virasoro algebra is the universal central extension of the Witt algebra and plays a significant role in the definition of the vertex operator algebra. The theory of vertex operator algebras has provided a mathematical foundation for conformal field theory; see [Lepowsky 2005]. Understanding such field theories in two dimensions involves problems about the representation theory of the Virasoro and vertex operator algebras.

The Witt algebra is an infinite-dimensional simple Lie algebra over $\mathbb{C}$ and is the smallest example of a Cartan-type Lie algebra. The Virasoro algebra has a triangular decomposition $\mathfrak{g}=\mathfrak{n}^{-} \oplus \mathfrak{h} \oplus \mathfrak{n}^{+}$, which allows one to define a Category $\mathbf{O}$. In this paper we study blocks of Category $\mathbb{O}$ for the Virasoro algebra, building on the foundational work of Fer̆gin and Fuchs [1990], who determined all maps between Verma modules for the Virasoro algebra. After making explicit the construction of BGG-resolutions for simple modules in these blocks (which is implicit in [Fer̆gin and Fuchs 1990]), we compute the $\mathfrak{n}^{+}$-cohomology with coefficients in any simple module. This extends results of Gončarova [1973a; 1973b], who calculated $\mathrm{H}^{\bullet}\left(\mathfrak{n}^{+}, \mathbb{C}\right)$, and of Rocha-Caridi and Wallach [1983a], who computed $\mathrm{H}^{\bullet}\left(\mathfrak{n}^{+}, L\right)$ for any simple module $L$ in the trivial block. This cohomological information allows us to calculate the extensions between simple and Verma modules. We then verify that our categories satisfy properties given in [Cline et al. 1997]; in particular, they have a KL theory. These properties yield a computation of extensions between all simple modules and imply that truncated blocks of Category 0 for the Virasoro algebra give rise to interesting Koszul algebras. We find it quite remarkable that KL theories naturally arise in the representation theory of the Virasoro algebra. It would be interesting to determine if this occurs in a more general context within the representation theory of Cartan-type Lie algebras.

The authors thank Brian Parshall for conversations about calculating extensions in quotient categories, Jonathan Kujawa for clarifying the connections between the extension theories used in Section 4.1, and the referee for several helpful comments and suggestions.

\section{Notation and preliminaries}

2.1. The Virasoro algebra is the Lie algebra $\mathfrak{g}=\mathbb{C}$-span $\left\{z, d_{k} \mid k \in \mathbb{Z}\right\}$ with bracket $[$,$] given by$

$\left[d_{k}, z\right]=0 \quad$ and $\quad\left[d_{j}, d_{k}\right]=(j-k) d_{j+k}+\frac{\delta_{j,-k}}{12}\left(j^{3}-j\right) z \quad$ for all $j, k \in \mathbb{Z}$. 
The Virasoro algebra can be decomposed into a direct sum of subalgebras $\mathfrak{g}=$ $\mathfrak{n}^{-} \oplus \mathfrak{h} \oplus \mathfrak{n}^{+}=\mathfrak{n}^{-} \oplus \mathfrak{b}^{+}$, where

$$
\mathfrak{n}^{-}=\mathbb{C}-\operatorname{span}\left\{d_{n} \mid n \in \mathbb{Z}_{<0}\right\}, \quad \mathfrak{h}=\mathbb{C}-\operatorname{span}\left\{d_{0}, z\right\}, \quad \mathfrak{n}^{+}=\mathbb{C}-\operatorname{span}\left\{d_{n} \mid n \in \mathbb{Z}_{>0}\right\},
$$

and $\mathfrak{b}^{+}=\mathfrak{h} \oplus \mathfrak{n}^{+}$. There is an antiinvolution $\sigma: \mathfrak{g} \rightarrow \mathfrak{g}$ given by $\sigma\left(d_{n}\right)=d_{-n}$ and $\sigma(z)=z$.

2.2. Category 0 and other categories. The Category $O$ consists of $\mathfrak{g}$-modules $M$ such that

- $M=\bigoplus_{\mu \in \mathfrak{h}^{*}} M^{\mu}$, where $\mathfrak{h}^{*}=\operatorname{Hom}_{\mathbb{C}}(\mathfrak{h}, \mathbb{C})$ and $M^{\mu}=\{m \in M \mid h m=$ $\mu(h) m$ for all $h \in \mathfrak{h}\}$;

- $M$ is finitely generated as a $\mathfrak{g}$-module;

- $M$ is $\mathfrak{n}^{+}$-locally finite.

This definition is more restricted than the one given in [Moody and Pianzola 1995]. Identify each integer $n \in \mathbb{Z}$ with a weight $n \in \mathfrak{h}^{*}$ by $n\left(d_{0}\right)=n$ and $n(z)=0$. Define a partial ordering on $\mathfrak{h}^{*}$ by

$$
\mu<\gamma \text { if } \mu=\gamma+n \text { for some } n \in \mathbb{Z}_{>0} .
$$

The category defined in [Moody and Pianzola 1995], which we denote $\tilde{0}$, consists of $\mathfrak{g}$-modules $M$ such that $M=\bigoplus_{\mu \in \mathfrak{h}^{*}} M^{\mu}$, $\operatorname{dim} M^{\mu}<\infty$, and there exist $\lambda_{1}, \ldots, \lambda_{n} \in \mathfrak{h}^{*}$ such that $M^{\mu} \neq 0$ only for $\mu \leq \lambda_{i}$ for some $i$. Then $\mathcal{O}$ (as we have defined it) is the full subcategory of $\tilde{0}$ consisting of finitely generated modules. Therefore, many of the results about $\tilde{O}$ proven in [Moody and Pianzola 1995] apply to 0 .

For $\mu \in \mathfrak{h}^{*}$, the Verma module $M(\mu)$ is the induced module

$$
M(\mu)=U(\mathfrak{g}) \otimes_{U\left(\mathfrak{b}^{+}\right)} \mathbb{C}_{\mu} .
$$

The Verma module $M(\mu)$ has a unique simple quotient, denoted $L(\mu)$. The modules $L(\mu)$ for $\mu \in \mathfrak{h}^{*}$ provide a complete set of simple modules in Category $\mathbb{0}$; see [Moody and Pianzola 1995, Section 2.3]. For $\mu, \gamma \in \mathfrak{h}^{*}$, define a partial ordering

$$
\mu \preceq \gamma \quad \text { if } L(\mu) \text { is a subquotient of } M(\gamma) .
$$

Extend this to an equivalence relation $\sim$. The blocks of $\mathfrak{g}$ are the equivalence classes of $\mathfrak{h}^{*}$ determined by $\sim$. For each block $[\mu] \subset \mathfrak{h}^{*}$, define $\mathbb{O}^{[\mu]}$ to be the full subcategory of 0 such that, for any $M \in \mathbb{O}^{[\mu]}$, the module $L(\gamma)$ is a subquotient of $M$ only for $\gamma \in[\mu]$. For $M \in \mathcal{O}, M=\bigoplus_{[\mu] \subset \mathfrak{h}^{*}} M^{[\mu]}$, where $M^{[\mu]} \in \mathbb{O}^{[\mu]}$; see [Moody and Pianzola 1995, 2.12.4]. 
We will use several other categories. Let $\mathcal{W}$ be the category whose objects are $\mathfrak{g}$-modules $M$ such that $M=\bigoplus_{\lambda \in \mathfrak{h}^{*}} M^{\lambda}$, where $M^{\lambda}$ is not necessarily finitedimensional. For a fixed weight $\mu \in \mathfrak{h}^{*}$, define $\mathcal{W}(\mu)$ to be the full subcategory of $\mathscr{W}$ whose objects are $\mathfrak{g}$-modules $M$ such that $M=\bigoplus_{\lambda \leq \mu} M^{\lambda}$.

The antiinvolution $\sigma$ can be used to define a duality functor $D$ on $\mathcal{W}$. For $M \in \mathscr{W}$, define $D M=\bigoplus_{\mu}\left(M^{\mu}\right)^{*}$ (as a vector space) with $\mathfrak{g}$-action given by $(x f)(v)=f(\sigma(x) v)$ for $x \in \mathfrak{g}, \quad f \in D M$, and $v \in M$. Then, $\operatorname{Hom}_{\mathscr{W}}\left(M, M^{\prime}\right) \cong$ $\operatorname{Hom}_{\mathscr{W}}\left(D M^{\prime}, D M\right)$ for all $M, M^{\prime} \in \mathcal{W}$. Since $\sigma(h)=h$ for $h \in \mathfrak{h}, D M$ decomposes as a direct sum of weight spaces where $(D M)^{\mu}=\left(M^{\mu}\right)^{*}$. Therefore, $\mathscr{W}(\mu)$ is closed under $D$. Finally, note that $D L \cong L$ for any simple module $L \in \mathbb{O}$.

\section{BGG resolutions and $\mathfrak{n}^{+}$-cohomology}

3.1. Theorem 1 describes all Verma module embeddings in a given block of $\mathcal{O}$. Since every nonzero map between Verma modules is an embedding, this describes all homomorphisms between Verma modules in a block. From this result one can construct BGG resolutions of the simple modules $L(\mu)$ to compute $\mathrm{H}^{\bullet}\left(\mathfrak{n}^{+}, L(\mu)\right)$.

Theorem 1 [Fer̆gin and Fuchs 1990, 1.9]. Suppose $\mu \in \mathfrak{h}^{*}$, and set $h=\mu\left(d_{0}\right)$ and $c=\mu(z) \in \mathbb{C}$. Define

$$
v=\frac{c-13+\sqrt{(c-1)(c-25)}}{12} \text { and } \beta=\sqrt{-4 v h+(v+1)^{2}},
$$

and consider the line in the rs-plane

$$
\mathscr{L}_{\mu}: r+v s+\beta=0 .
$$

The Verma module embeddings involving $M(\mu)$ are determined by integer points $(r, s)$ on $\mathscr{L}_{\mu}$ :

(i) Suppose $\mathscr{L}_{\mu}$ passes through no integer points or one integer point $(r, s)$ with $r s=0$. Then the block $[\mu]$ is given by $[\mu]=\{\mu\}$.

(ii) Suppose $\mathscr{L}_{\mu}$ passes through exactly one integer point $(r, s)$ with $r s \neq 0$. The block $[\mu]$ is given by $[\mu]=\{\mu, \mu+r s\}$. The block structure is given below, where an arrow $\lambda \rightarrow \gamma$ between weights indicates $M(\lambda) \subseteq M(\gamma)$.

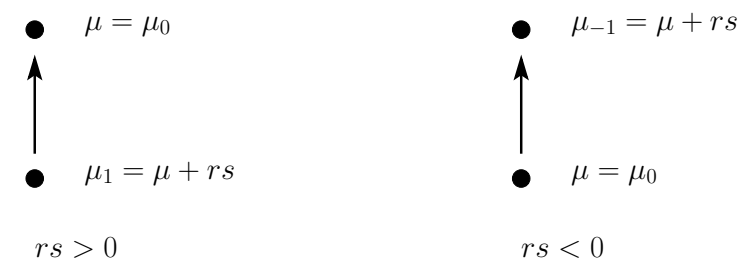




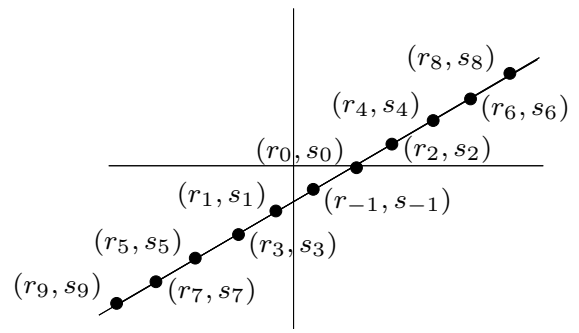

Figure 1. The points $(r, s)$ on $\mathscr{L}_{\mu}$.

(iii) Suppose $\mathscr{L}_{\mu}$ passes through infinitely many integer points and crosses an axis at an integer point. Label these points $\left(r_{i}, s_{i}\right)$ so that

$$
\cdots<r_{-2} s_{-2}<r_{-1} s_{-1}<0=r_{0} s_{0}<r_{1} s_{1}<r_{2} s_{2}<\cdots
$$

as in Figure 1. The block $[\mu]$ is given by $[\mu]=\left\{\mu_{i}=\mu+r_{i} s_{i}\right\}$. The block structure is given below.

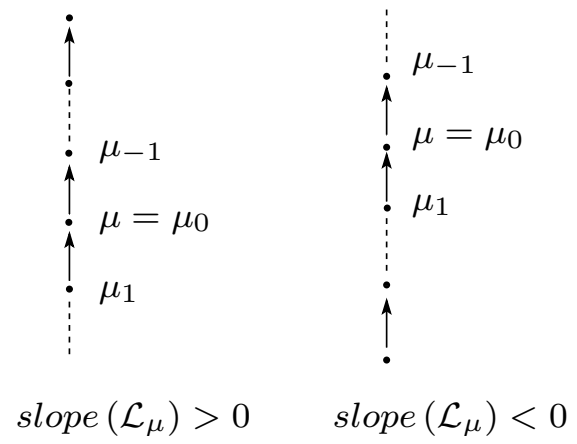

(iv) Suppose $\mathscr{L}_{\mu}$ passes through infinitely many integer points and does not cross either axis at an integer point. Label the integer points $\left(r_{i}, s_{i}\right)$ on $\mathscr{L}_{\mu}$ so that

$$
\cdots<r_{-1} s_{-1}<r_{0} s_{0}<0<r_{1} s_{1}<r_{2} s_{2}<\cdots .
$$

Also consider the auxiliary line $\widetilde{\mathscr{L}}_{\mu}$ with the same slope as $\mathscr{L}_{\mu}$ passing through the point $\left(-r_{1}, s_{1}\right)$. Label the integer points on this line $\left(r_{j}^{\prime}, s_{j}^{\prime}\right)$ in the same way as $\mathscr{L}_{\mu}$. The block $[\mu]$ is given by $[\mu]=\left\{\mu_{i}, \mu_{i}^{\prime}\right\}$, where

$$
\mu_{i}=\left\{\begin{array}{ll}
\mu+r_{i} s_{i} & \text { for } i \text { odd }, \\
\mu+r_{1} s_{1}+r_{i}^{\prime} s_{i}^{\prime} & \text { for } \text { i even, }
\end{array} \quad \mu_{i}^{\prime}= \begin{cases}\mu+r_{i+1} s_{i+1} & \text { for } i \text { odd }, \\
\mu+r_{1} s_{1}+r_{i+1}^{\prime} s_{i+1}^{\prime} & \text { for } i \text { even } .\end{cases}\right.
$$

The block structure is given below. 

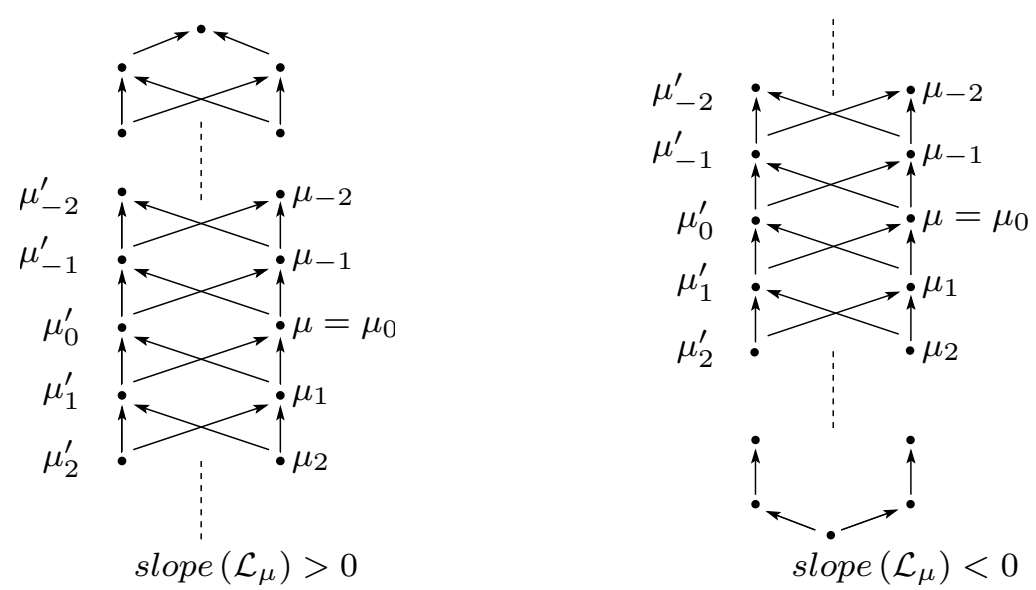

We will refer to blocks as in case (iii) as "thin" blocks and blocks as in case (iv) as "thick" blocks. The second type of thick block has a highest weight poset structure equivalent to the Bruhat order on $D_{\infty}$, the infinite dihedral group.

3.2. BGG resolutions. Fer̆gin and Fuchs [1988] observe without elaboration that their result, Theorem 1 above, yields a BGG resolution for the simple modules in Category 0 . We now provide the details for constructing these resolutions.

Given a module $M$ in Category $O$, define the radical of $M, \operatorname{rad} M$, to be the smallest submodule such that $M / \operatorname{rad} M$ is semisimple. $\operatorname{Put} \operatorname{rad}^{0} M=M$, and for $i>0$ put $\operatorname{rad}^{i} M=\operatorname{rad}\left(\operatorname{rad}^{i-1} M\right)$. This defines a decreasing filtration of $M$, the radical filtration. For $i \geq 0$, layer $i$ of the radical filtration is defined to be $\operatorname{rad}_{i} M=\operatorname{rad}^{i} M / \operatorname{rad}^{i+1} M$. We also write hd $M=M / \operatorname{rad} M$. In general, see [Fergin and Fuchs 1990], the terms and the layers of the radical filtration of $M(\mu)$, in the notation of Theorem 1(ii)-(iv), are as follows:

$$
\operatorname{rad}_{0} M(\mu)=L(\mu)
$$

$$
\begin{array}{ll}
\operatorname{rad}^{i} M(\mu)=M\left(\mu_{i}\right)+M\left(\mu_{i}^{\prime}\right) & \text { for } i>0, \\
\operatorname{rad}_{i} M(\mu)=L\left(\mu_{i}\right) \oplus L\left(\mu_{i}^{\prime}\right) & \text { for } i>0 .
\end{array}
$$

If $\mu$ belongs to a finite or thin block, then terms involving $\mu_{i}^{\prime}$ are to be ignored. Also if $\mu$ belongs to a block with a minimal element, say $\mu_{n}$, then $\operatorname{rad}^{n} M(\mu)=\operatorname{rad}_{n} M(\mu)=M\left(\mu_{n}\right)=L\left(\mu_{n}\right), \operatorname{rad}^{i} M(\mu)=\operatorname{rad}_{i} M(\mu)=0$ for $i>n$.

Assume $\mu$ belongs to a thick block. According to [Bernšteĭn et al. 1975], there will be a complex $C_{\bullet} \rightarrow L(\mu) \rightarrow 0$, where $C_{i}$ is the direct sum of the Verma modules $M\left(\mu_{i}\right) \oplus M\left(\mu_{i}^{\prime}\right)$, provided that to each edge of the poset below $\mu$, using the ordering $\preceq$, it is possible to assign a sign +1 or -1 so that the product of the signs on any diamond is -1 . Such a labeling is indicated in Figure 2. It is easy to check directly in this case that the resulting complex is in fact a resolution, called a BGG resolution of $L(\mu)$. 

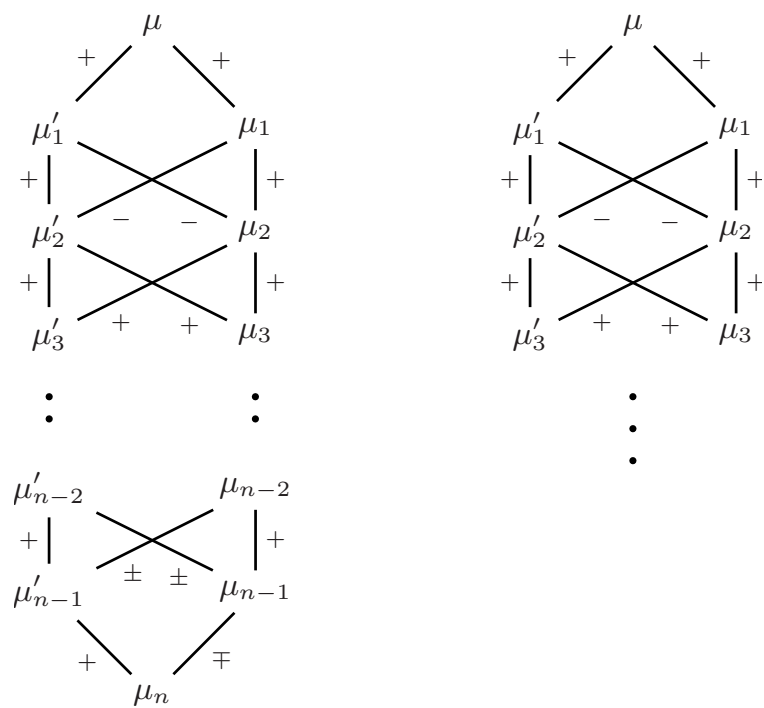

Figure 2. Assignment of signs.

Explicitly, the following are BGG resolutions of $L(\mu)$ : for $\mu$ belonging to a thick block with a minimal element $\mu_{n}$,

$$
\begin{aligned}
0 \rightarrow M\left(\mu_{n}\right) \rightarrow M\left(\mu_{n-1}\right) \oplus M\left(\mu_{n-1}^{\prime}\right) & \rightarrow \cdots \\
& \rightarrow M\left(\mu_{1}\right) \oplus M\left(\mu_{1}^{\prime}\right) \rightarrow M(\mu) \rightarrow L(\mu) \rightarrow 0 ;
\end{aligned}
$$

and for $\mu$ belonging to a thick block with a maximal element,

$$
\cdots \rightarrow M\left(\mu_{i}\right) \oplus M\left(\mu_{i}^{\prime}\right) \rightarrow \cdots \rightarrow M\left(\mu_{1}\right) \oplus M\left(\mu_{1}^{\prime}\right) \rightarrow M(\mu) \rightarrow L(\mu) \rightarrow 0 .
$$

Next consider a weight $\mu \in \mathfrak{h}^{*}$ belonging to a thin block or a finite block. Then $\operatorname{rad} M(\mu)=M\left(\mu_{1}\right)$ if $\mu_{1}$ exists in the block, and $\operatorname{rad} M(\mu)=0$ otherwise. Thus, if $M(\mu)$ is not itself irreducible, the BGG resolution of $L(\mu)$ is

$$
0 \rightarrow M\left(\mu_{1}\right) \rightarrow M(\mu) \rightarrow L(\mu) \rightarrow 0 .
$$

We now introduce some additional notation. Fix $\mu \in \mathfrak{h}^{*}$. Define a length function $l:[\mu] \rightarrow \mathbb{Z}$ by

$$
l\left(\mu_{i}\right)=l\left(\mu_{i}^{\prime}\right)=i,
$$

using the notation of Theorem 1(ii)-(iv). While $l($ ) depends on a choice of representative $\mu$ for the block, the value (and, in particular, the parity) of $l(v)-l(\gamma)$ for $\nu, \gamma \in[\mu]$ is independent of the choice of representative. This will be relevant later. 
In summary of the description given above, each simple module $L(\mu)$ has a BGG resolution $\cdots \rightarrow C_{1} \rightarrow C_{0} \rightarrow L(\mu) \rightarrow 0$ where

$$
C_{i}= \begin{cases}\bigoplus_{l(v)=i, v \leq \mu} M(v) & \text { if }[\mu] \text { is a thick block or a finite block, } \\ \bigoplus_{l(v)=i} M(v) & \text { if } i \leq 1 \text { and }[\mu] \text { is a thin block, } \\ 0 & \text { if } i>1 \text { and }[\mu] \text { is a thin block. }\end{cases}
$$

3.3. $\mathfrak{n}^{+}$-cohomology. Gončarova $[1973 a ; 1973 b]$ proved that

$$
\mathrm{H}^{k}\left(\mathfrak{n}^{+}, \mathbb{C}\right)=\mathbb{C}_{\left(3 k^{2}+k\right) / 2} \oplus \mathbb{C}_{\left(3 k^{2}-k\right) / 2}
$$

Rocha-Caridi and Wallach use Gončarova's work to obtain BGG resolutions for $\mathbb{C}$ [1983a] and for the other simple modules in the trivial block [1983b]. Using this, they compute $\mathrm{H}^{k}\left(\mathfrak{n}^{+}, L\right)$ for any simple module $L$ in the trivial block [1983b]. We extend their result to cohomology with coefficients in any simple module in $\mathbb{O}$.

Theorem 2. Let $\mu \in \mathfrak{h}^{*}$, and let $k \in \mathbb{Z}_{\geq 0}$.

(a) Suppose that $\mu$ belongs to a thick block or a finite block. As an $\mathfrak{h}$-module,

$$
\mathrm{H}^{k}\left(\mathfrak{n}^{+}, L(\mu)\right) \cong \mathrm{H}_{k}\left(\mathfrak{n}^{-}, L(\mu)\right) \cong \bigoplus_{\substack{v \in[\mu], \nu \preceq \mu \\ l(\nu)-l(\mu)=k}} \mathbb{C}_{\nu} .
$$

(b) Suppose that $\mu$ belongs to a thin block. As an $\mathfrak{h}$-module,

$$
\mathrm{H}^{k}\left(\mathfrak{n}^{+}, L(\mu)\right) \cong \mathrm{H}_{k}\left(\mathfrak{n}^{-}, L(\mu)\right) \cong\left\{\begin{array}{cl}
\bigoplus_{v \in[\mu], v \leq \mu} \mathbb{C}_{v} & \text { if } k \leq 1, \\
l(\nu)-l(\mu)=k & \\
0 & \text { if } k>1 .
\end{array}\right.
$$

Proof. We first compute the homology groups $\mathrm{H}_{k}\left(\mathfrak{n}^{-}, L(\mu)\right)$, where $\mathrm{H}_{k}\left(\mathfrak{n}^{-},-\right)$is the $k$-th left derived functor of $\mathbb{C} \otimes_{U\left(\mathfrak{n}^{-}\right)}-$. Since Verma modules are free $U\left(\mathfrak{n}^{-}\right)$modules, apply $\mathbb{C} \otimes_{U\left(\mathfrak{n}^{-}\right)}-$to the resolution (5). Note that $\mathbb{C} \otimes_{U\left(\mathfrak{n}^{-}\right)} M(v) \cong \mathbb{C}_{v}$. The resulting differential maps in the resolution are $\mathfrak{h}$-equivariant, and $\mathbb{C}_{v}$ appears at most once in the resolution for each weight $v$. Therefore, all of the differential maps must be zero. This verifies that $\mathrm{H}_{k}\left(\mathfrak{n}^{-}, L(\mu)\right)$ is as stated.

We now show that $\mathrm{H}_{k}\left(\mathfrak{n}^{-}, L(\mu)\right) \cong \mathrm{H}^{k}\left(\mathfrak{n}^{+}, L(\mu)\right)$. This may be well known; it is claimed in [Rocha-Caridi and Wallach 1983a] to follow from "standard arguments." Because the infinite-dimensional case seems somewhat subtle, we include a proof for completeness.

Write $L=L(\mu)$. Recall that $\mathrm{H}_{k}\left(\mathfrak{n}^{-}, L\right)$ can be computed using the complex

$$
\cdots \rightarrow \Lambda^{k}\left(\mathfrak{n}^{-}\right) \otimes L \stackrel{d_{k}}{\rightarrow} \Lambda^{k-1}\left(\mathfrak{n}^{-}\right) \otimes L \rightarrow \cdots
$$


and $\mathrm{H}^{k}\left(\mathfrak{n}^{+}, L\right)$ can be computed using the complex

$$
\cdots \rightarrow \Lambda^{k}\left(\left(\mathfrak{n}^{+}\right)^{*}\right) \otimes L \stackrel{d^{k}}{\rightarrow} \Lambda^{k+1}\left(\left(\mathfrak{n}^{+}\right)^{*}\right) \otimes L \rightarrow \cdots .
$$

We extend the notion of duality defined in Section 2.2 to $\Lambda^{k}\left(\mathfrak{n}^{-}\right)$, viewed as an $\mathfrak{h}$-module, as follows. For $f \in \oplus_{\lambda \in \mathfrak{h}^{*}}\left(\left(\Lambda^{k}\left(\mathfrak{n}^{-}\right)\right)^{*}\right)^{\lambda}$, define $\tilde{f} \in\left(\Lambda^{k}\left(\mathfrak{n}^{+}\right)\right)^{*}$ by $\tilde{f}(x)=f(\sigma(x))$ for $x \in \Lambda^{k}\left(\mathfrak{n}^{+}\right)$. Let $D\left(\Lambda^{k}\left(\mathfrak{n}^{-}\right)\right)=\left\{\tilde{f} \mid f \in \oplus_{\lambda \in \mathfrak{h}^{*}}\left(\left(\Lambda^{k}\left(\mathfrak{n}^{-}\right)\right)^{*}\right)^{\lambda}\right\}$. Define $\Lambda^{k}\left(D\left(\mathfrak{n}^{-}\right)\right) \subseteq \Lambda^{k}\left(\left(\mathfrak{n}^{+}\right)^{*}\right)$ analogously.

By choosing a basis for each weight space $\left(\Lambda^{k}\left(\mathfrak{n}^{-}\right) \otimes L\right)^{\lambda}$, we can construct an $\mathfrak{h}$-module embedding $\Lambda^{k}\left(\mathfrak{n}^{-}\right) \otimes L \rightarrow D\left(\Lambda^{k}\left(\mathfrak{n}^{-}\right)\right) \otimes L \subseteq\left(\Lambda^{k}\left(\mathfrak{n}^{+}\right)\right)^{*} \otimes L$. Since $D(L) \cong L$, the differential map $d_{k+1}$ induces a codifferential map $\tilde{d}_{k}: D\left(\Lambda^{k}\left(\mathfrak{n}^{-}\right)\right) \otimes$ $L \rightarrow D\left(\Lambda^{k+1}\left(\mathfrak{n}^{-}\right)\right) \otimes L$ as follows. Let $\left.f \in \oplus_{\lambda \in \mathfrak{h}^{*}}\left(\Lambda^{k}\left(\mathfrak{n}^{-}\right)\right)^{*}\right)^{\lambda}$ and $g \in \oplus_{\lambda \in \mathfrak{h}^{*}}\left(L^{*}\right)^{\lambda}$. Then $f \otimes g$ corresponds to an element $\widetilde{f \otimes g} \in D\left(\Lambda^{k}\left(\mathfrak{n}^{-}\right)\right) \otimes D L \cong D\left(\Lambda^{k}\left(\mathfrak{n}^{-}\right)\right) \otimes L$. Define $\tilde{d}_{k}(\widetilde{f \otimes g})(x \otimes m)=(f \otimes g)\left(d_{k+1}(\sigma(x) \otimes m)\right)$ for $x \in \Lambda^{k+1}\left(\mathfrak{n}^{+}\right)$and $m \in L$. For $\lambda \in \mathfrak{h}^{*}$, it can be shown that $\operatorname{dim}\left(\operatorname{ker} \tilde{d}_{k}\right)^{\lambda}-\operatorname{dim}\left(\operatorname{Im} \tilde{d}_{k-1}\right)^{\lambda}=\operatorname{dim}\left(\operatorname{ker} d_{k}\right)^{\lambda}-$ $\operatorname{dim}\left(\operatorname{Im} d_{k+1}\right)^{\lambda}$. This implies

$$
\mathrm{H}^{k}\left(D\left(\Lambda^{\bullet}\left(\mathfrak{n}^{-}\right)\right) \otimes L\right) \cong \mathrm{H}_{k}\left(\mathfrak{n}^{-}, L\right) .
$$

For each $k$, define $\phi_{k}=\phi: \Lambda^{k}\left(\left(\mathfrak{n}^{+}\right)^{*}\right) \rightarrow\left(\Lambda^{k}\left(\mathfrak{n}^{+}\right)\right)^{*}$ by

$$
\phi\left(f_{1} \wedge \cdots \wedge f_{k}\right)\left(x_{1} \wedge \cdots \wedge x_{k}\right)=\sum_{\tau \in S_{k}} \operatorname{sgn}(\tau) \prod_{i} f_{i}\left(x_{\tau(i)}\right)
$$

for $x_{i} \in \mathfrak{n}^{+}$and $f_{i} \in\left(\mathfrak{n}^{+}\right)^{*}$. The map $\phi$ is an $\mathfrak{h}$-module isomorphism and satisfies $\phi\left(\Lambda^{k}\left(D\left(\mathfrak{n}^{-}\right)\right)\right)=D\left(\Lambda^{k}\left(\mathfrak{n}^{-}\right)\right)$. Moreover, it can be checked that $\left(\phi_{k+1} \otimes 1\right) \circ d^{k}=$ $\tilde{d}_{k} \circ\left(\phi_{k} \otimes 1\right)$ on $D\left(\Lambda^{k}\left(\mathfrak{n}^{-}\right)\right) \otimes L$. Therefore, $\phi \otimes 1$ gives an isomorphism

$$
\mathrm{H}^{k}\left(D\left(\Lambda^{\bullet}\left(\mathfrak{n}^{-}\right)\right) \otimes L\right) \cong \mathrm{H}^{k}\left(\Lambda^{\bullet}\left(D\left(\mathfrak{n}^{-}\right)\right) \otimes L\right) .
$$

To complete the proof, we need to show that $\mathrm{H}^{k}\left(\Lambda^{\bullet}\left(D\left(\mathfrak{n}^{-}\right)\right) \otimes L\right) \cong \mathrm{H}^{k}\left(\mathfrak{n}^{+}, L\right)$, which entails checking that

(i) for $X \in \operatorname{Im}\left(d^{k-1}\right) \cap \Lambda^{k}\left(D\left(\mathfrak{n}^{-}\right)\right) \otimes L$, there is a $Y \in \Lambda^{k-1}\left(D\left(\mathfrak{n}^{-}\right)\right) \otimes L$ with $d^{k-1}(Y)=X$

(ii) for $X \in \operatorname{ker}\left(d^{k}\right)$, there is an $\tilde{X} \in \Lambda^{k}\left(D\left(\mathfrak{n}^{-}\right)\right) \otimes L$ such that $X-\tilde{X} \in \operatorname{Im}\left(d^{k-1}\right)$.

Let $X \in \Lambda^{k}\left(\left(\mathfrak{n}^{+}\right)^{*}\right) \otimes L$. The space $\Lambda^{k}\left(\left(\mathfrak{n}^{+}\right)^{*}\right) \otimes L$ decomposes as a direct product of its weight spaces. Write $X=\prod_{\lambda \in \mathfrak{h}^{*}} X_{\lambda}$, where $X_{\lambda} \in\left(\Lambda^{k}\left(\left(\mathfrak{n}^{+}\right)^{*}\right) \otimes L\right)^{\lambda}$. Then $X_{\lambda}=f_{1} \otimes m_{1}+\cdots+f_{n} \otimes m_{n}$ for some $f_{i} \in \Lambda^{k}\left(\left(\mathfrak{n}^{+}\right)^{*}\right)^{\mu_{i}}$ and $m_{i} \in L^{v_{i}}$ with $\mu_{i}+v_{i}=\lambda$. From the definition of $\Lambda^{k}\left(D\left(\mathfrak{n}^{-}\right)\right)$, we see that $\Lambda^{k}\left(D\left(\mathfrak{n}^{-}\right)\right)$is the set of elements $f \in \Lambda^{k}\left(\left(\mathfrak{n}^{+}\right)^{*}\right)$ with $f=\prod_{\mu \in \mathfrak{h}^{*}} f_{\mu}$ such that $f_{\mu} \neq 0$ for only finitely many $\mu$. Therefore, $f_{i} \in \Lambda^{k}\left(D\left(\mathfrak{n}^{-}\right)\right)$, and so $X_{\lambda} \in \Lambda^{k}\left(D\left(\mathfrak{n}^{-}\right)\right) \otimes L$.

The differential map $d^{k}$ preserves weight spaces. Suppose $X=d^{k-1}(\tilde{Y})$ for some $\tilde{Y} \in \Lambda^{k-1}\left(\left(\mathfrak{n}^{+}\right)^{*}\right) \otimes L$. Define $Y=\prod_{\lambda \in \mathfrak{h}^{*}} Y_{\lambda}$ by $Y_{\lambda}=\tilde{Y}_{\lambda}$ if $X_{\lambda} \neq 0$ and 
$Y_{\lambda}=0$ otherwise. If $X \in \Lambda^{k}\left(D\left(\mathfrak{n}^{-}\right)\right) \otimes L$ then $Y \in \Lambda^{k-1}\left(D\left(\mathfrak{n}^{-}\right)\right) \otimes L$. This proves (i).

Now let $X \in \operatorname{ker}\left(d^{k}\right)$. Then $d^{k}\left(X_{\lambda}\right)=0$ for all $\lambda \in \mathfrak{h}^{*}$. Equations (7) and (8) imply that $\mathrm{H}^{k}\left(\Lambda^{\bullet}\left(D\left(\mathfrak{n}^{-}\right)\right) \otimes L\right)$ is finite dimensional. Because $X_{\lambda} \in \Lambda^{k}\left(D\left(\mathfrak{n}^{-}\right)\right) \otimes L$, this means that there are only finitely many $\lambda_{1}, \ldots, \lambda_{r}$ such that $X_{\lambda_{i}} \neq 0$ and $X_{\lambda_{i}} \neq d^{k}\left(Y_{\lambda_{i}}\right)$ for some $Y_{\lambda_{i}} \in \Lambda^{k-1}\left(D\left(\mathfrak{n}^{-}\right)\right) \otimes L$. Define $\tilde{X}_{\lambda_{i}}=X_{\lambda_{i}}$ for $i=1, \ldots, r$ and $\tilde{X}_{\lambda}=0$ otherwise. Then $\tilde{X} \in\left(\Lambda^{k}\left(D\left(\mathfrak{n}^{-}\right)\right) \otimes L\right.$ and $X-\tilde{X} \in \operatorname{Im}\left(d^{k-1}\right)$. This proves (ii).

Corollary 3. Every irreducible module in Category 0 for the Virasoro algebra is a Kostant module (in the sense of [Boe and Hunziker 2006]).

\section{Extensions}

The structure of the infinite blocks of 0 presents various obstacles in computing Ext-groups. The infinite blocks with a minimal element do not have enough projectives. In the infinite blocks with a maximal element, objects do not generally have finite length. We demonstrate that the first problem can be remedied by truncation, and the second can be addressed via a quotient construction.

4.1. Cohomology and truncated categories. We first define the truncation of an infinite block of $\mathcal{O}$ having a minimal element. Fix a weight $\mu$ in the block $\mathscr{C}$, and consider the full subcategory $\mathscr{b}(\mu)$ - called the truncation of the block at $\mu$ - of modules all of whose composition factors have highest weights less than or equal to $\mu$, using the partial ordering given in (2).

Now let $\mathscr{C}$ be a finite block of $\mathscr{O}$, an infinite block of $\mathcal{O}$ with a maximal element, or a truncated infinite block with minimal element. Denote the weight poset of $\mathscr{C}$ by $\Lambda$. Then there is a maximal element $\mu \in \Lambda$. If $\mathscr{C}$ is a truncated thick block, we can write $\mu=\mu_{0}$ as in Theorem 1(iv). We assume that $\mu$ is chosen so that $\mu_{0} \leq \mu_{0}^{\prime}$ in the partial ordering given by Equation (1). Then $\Lambda=\{v \in[\mu] \mid v \leq \mu\}$, which allows us to compare $\mathscr{C}$ and $\mathscr{W}(\mu)$. We now use Theorem 2 to compute $\operatorname{Ext}_{\mathscr{C}}^{i}(M(\lambda), L(v))$ by passing through relative cohomology and using the categories $\mathscr{W}$ and $\mathscr{W}(\mu)$ defined in Section 2.2.

Lemma 4. If $\lambda, v \in \Lambda$ and $i \in \mathbb{Z}_{\geq 0}$, then $\operatorname{Ext}_{W}^{i}(M(\lambda), L(v)) \cong \mathrm{H}^{i}\left(\mathfrak{n}^{+}, L(v)\right)^{\lambda}$.

Proof. For $i \in \mathbb{Z}_{\geq 0}$, define $P_{i}=U(\mathfrak{g}) \otimes_{U(\mathfrak{h})} \Lambda^{i}(\mathfrak{g} / \mathfrak{h})$. Then, for any $\mathfrak{g}$-module $M$, the sequence (with suitably defined maps) $\cdots \rightarrow P_{2} \otimes_{\mathbb{C}} M \rightarrow P_{1} \otimes_{\mathbb{C}} M \rightarrow$ $M \rightarrow 0$ is a $(\mathfrak{g}, \mathfrak{h})$-projective resolution [Kumar 2002, 3.1.8]. If $M=M(\lambda)$, then 
$P_{i} \otimes_{\mathbb{C}} M(\lambda) \in \mathscr{W}$, and so this gives a projective resolution in $\mathcal{W}$. We then have

$$
\begin{aligned}
\operatorname{Ext}_{W}^{i}(M(\lambda), L(v)) & \cong \operatorname{Ext}_{(\mathfrak{g}, \mathfrak{h})}^{i}(M(\lambda), L(v)) \cong \operatorname{Ext}_{\left(\mathfrak{b}^{+}, \mathfrak{h}\right)}^{i}\left(\mathbb{C}_{\lambda}, L(v)\right) \\
& \cong \operatorname{Ext}_{\left(\mathfrak{b}^{+}, \mathfrak{h}\right)}^{i}\left(\mathbb{C}, \mathbb{C}_{-\lambda} \otimes L(v)\right) \cong \mathrm{H}^{i}\left(\mathfrak{b}^{+}, \mathfrak{h}, \mathbb{C}_{-\lambda} \otimes L(v)\right) \\
& \cong \mathrm{H}^{i}\left(\mathfrak{n}^{+}, L(v)\right)^{\lambda} .
\end{aligned}
$$

The second through fourth isomorphisms follow from [Kumar 2002, 3.1.14, 3.1.13, 3.9], respectively. The last isomorphism follows from definitions.

There are two functors $\eta: \mathscr{W} \rightarrow \mathscr{W}(\mu)$ and $\theta: \mathscr{W} \rightarrow \mathscr{W}(\mu)$ defined as follows. For $M \in \mathscr{W}$, there is a unique minimal submodule $M^{\prime} \subseteq M$ such that $M / M^{\prime} \in \mathcal{W}(\mu)$. Define $\eta M=M / M^{\prime}$. Note that $M^{\prime}$ is generated, as a $\mathfrak{g}$-module, by $\bigoplus_{\lambda \neq \mu} M^{\lambda}$. Then, for any $N, M \in \mathcal{W}$, and any $\mathfrak{g}$-module homomorphism $f: M \rightarrow N$ we have $f\left(M^{\prime}\right) \subseteq N^{\prime}$. Therefore $f$ induces a homomorphism from $\eta M$ to $\eta N$. Define $\eta(f)$ to be this map. For $M \in \mathcal{W}$, there is also a unique maximal submodule $M^{\prime \prime}$ such that $M^{\prime \prime} \in \mathcal{W}(\mu)$. Define $\theta M=M^{\prime \prime}$. For any $N, M \in \mathcal{W}$ and any $\mathfrak{g}$-module homomorphism $f: M \rightarrow N$, define $\theta(g)=\left.g\right|_{M^{\prime \prime}}$. Using these functors we relate $\operatorname{Ext}_{W}^{i}(-,-)$ and $\operatorname{Ext}_{W(\mu)}^{i}(-,-)$.

Lemma 5. Let $M, N \in \mathcal{W}(\mu)$. Then $\operatorname{Ext}_{\mathcal{W}(\mu)}^{i}(M, N)=\operatorname{Ext}_{\mathscr{W}}^{i}(M, N)$.

Proof. First observe that $\eta$ takes projectives to projectives and $\theta$ takes injectives to injectives. Let $N \rightarrow I_{\bullet}$ be an injective resolution in $\mathscr{W}$. Since $M \in \mathscr{W}(\mu)$, $\operatorname{Hom}_{\mathfrak{g}}\left(M, I_{k}\right) \cong \operatorname{Hom}_{\mathfrak{g}}\left(M, \theta I_{k}\right)$. Therefore, $\operatorname{Ext}_{\mathfrak{W}}^{i}(M, N)=\mathrm{H}^{i}\left(\operatorname{Hom}_{\mathfrak{g}}\left(M, I_{k}\right)\right) \cong$ $\mathrm{H}^{i}\left(\operatorname{Hom}_{\mathfrak{g}}\left(M, \theta I_{k}\right)\right)$. The lemma follows if we can show that $\theta$ is acyclic on $N$ because this would imply that $N=\theta N \rightarrow \theta I_{\bullet}$ is an injective resolution.

Note that $\mathrm{H}^{i}\left(\theta I_{\bullet}\right)=0$ if and only if $\mathrm{H}^{i}\left(\left(\theta I_{\bullet}\right)^{\gamma}\right)=0$ for all $\gamma \in \mathfrak{h}^{*}$. For $\gamma \in \mathfrak{h}^{*}$, define $P_{\gamma}=U(\mathfrak{g}) \otimes_{U(\mathfrak{h})} \mathbb{C}_{\gamma}$. Then $\left(\theta I_{k}\right)^{\gamma}=\operatorname{Hom}_{\mathfrak{g}}\left(P_{\gamma}, \theta I_{k}\right)$. This implies

$$
\begin{aligned}
\mathrm{H}^{i}\left(\left(\theta I_{\bullet}\right)^{\gamma}\right) & \cong \mathrm{H}^{i}\left(\operatorname{Hom}_{\mathfrak{g}}\left(P_{\gamma}, \theta I_{\bullet}\right)\right) \\
& \cong \mathrm{H}^{i}\left(\operatorname{Hom}_{\mathfrak{g}}\left(\eta P_{\gamma}, I_{\bullet}\right)\right) \cong \operatorname{Ext}_{\mathscr{W}}^{i}\left(\eta P_{\gamma}, N\right) .
\end{aligned}
$$

Therefore, to complete the proof it is enough to show that $\operatorname{Ext}_{w}^{i}\left(\eta P_{\gamma}, N\right)=0$ for $i \geq 1$. There is a short exact sequence $0 \rightarrow P_{\gamma}^{\prime} \rightarrow P_{\gamma} \rightarrow \eta P_{\gamma} \rightarrow 0$, which gives a long exact sequence

$$
\cdots \rightarrow \operatorname{Ext}_{W}^{i-1}\left(P_{\gamma}^{\prime}, N\right) \rightarrow \operatorname{Ext}_{W}^{i}\left(\eta P_{\gamma}, N\right) \rightarrow \operatorname{Ext}_{w}^{i}\left(P_{\gamma}, N\right) \rightarrow \cdots
$$

Since $P_{\gamma}$ is projective in $\mathcal{W}, \operatorname{Ext}_{\mathscr{W}}^{i}\left(P_{\gamma}, N\right)=0$ for $i \geq 1$. We claim $\operatorname{Ext}_{\mathscr{W}}^{i-1}\left(P_{\gamma}^{\prime}, N\right)=$ 0 for all $i$. To see this, let

$P_{\gamma}^{\not \mu}=\operatorname{span}\left\{m \in P_{\gamma} \mid m \in P_{\gamma}^{v}\right.$ for some $v \not \leq \mu, m=x \otimes 1$ for some $\left.x \in U\left(\mathfrak{n}^{+}\right)\right\}$.

Then $P_{\gamma}^{ \pm \mu}$ is a $\mathfrak{b}^{+}$-module. 
Define $\mathscr{W}_{\mathfrak{b}^{+}}$to be the category of $\mathfrak{b}^{+}$-modules $M$ such that $M=\bigoplus_{\lambda \in \mathfrak{h}^{*}} M^{\lambda}$. Define $Q_{k}=U\left(\mathfrak{b}^{+}\right) \otimes_{U(\mathfrak{h})} \Lambda^{k}\left(\mathfrak{b}^{+} / \mathfrak{h}\right) \otimes_{\mathbb{C}} P_{\gamma}^{ \pm \mu}$. Then $Q_{\bullet} \rightarrow P_{\gamma}^{ \pm \mu}$ is a projective resolution of $P_{\gamma}^{ \pm \mu}$ in $\mathscr{W}_{\mathfrak{b}^{+}}$; see [Kumar 2002, 3.1.8]. Also, $U(\mathfrak{g}) \otimes_{U\left(\mathfrak{b}^{+}\right)} Q$. is a projective resolution of $P_{\gamma}^{\prime} \cong U(\mathfrak{g}) \otimes_{U\left(\mathfrak{b}^{+}\right)} P_{\gamma}^{ \pm \mu}$ in $\mathcal{W}$. Moreover, $\operatorname{Hom}_{\mathfrak{g}}\left(U(\mathfrak{g}) \otimes_{U\left(\mathfrak{b}^{+}\right)}\right.$ $\left.Q_{k}, N\right)=\operatorname{Hom}_{\mathfrak{b}^{+}}\left(Q_{k}, N\right)=0$ since $Q_{k}^{v} \neq 0$ only for $v \not \leq \mu$ and $N^{v} \neq 0$ only for $v \leq \mu$. Therefore $\operatorname{Ext}_{\mathscr{W}}^{i-1}\left(P_{\gamma}^{\prime}, N\right)=0$ for all $i$. This implies that $\operatorname{Ext}_{\mathscr{W}}^{i}\left(\eta P_{\gamma}, N\right)=0$ for $i \geq 1$.

We now transfer the information from $\mathcal{W}(\mu)$ to $\mathscr{C}$.

Theorem 6. Let $\mathscr{C}$ be a finite block of $\mathcal{O}$, an infinite block of $\mathbb{O}$ with a maximal element, or a truncated infinite block with minimal element. Let $\Lambda$ be the weight poset of $\mathscr{C}$ with maximal element $\mu$, and let $\lambda, v \in \Lambda$. Then for $i \geq 0$,

(a) if $\mathscr{C}$ is a thick block or a finite block,

$$
\operatorname{Ext}_{\mathscr{C}}^{i}(M(\lambda), L(v)) \cong \begin{cases}\mathbb{C} & \text { if } \lambda \preceq v \text { and } l(\lambda)-l(v)=i, \\ 0 & \text { otherwise }\end{cases}
$$

(b) if $\mathscr{C}$ is a thin block,

$$
\operatorname{Ext}_{\mathscr{C}}^{i}(M(\lambda), L(v)) \cong \begin{cases}\mathbb{C} & \text { if } \lambda \preceq v \text { and } l(\lambda)-l(v)=i \text { for } i \leq 1, \\ 0 & \text { otherwise. }\end{cases}
$$

Proof. Let $M, N \in \mathscr{C}$. Given Theorem 2 and Lemmas 4 and 5, it is enough to show that $\operatorname{Ext}_{\mathscr{C}}^{i}(M, N) \cong \operatorname{Ext}_{\mathscr{W}(\mu)}^{i}(M, N)$.

Let $\gamma \in \mathfrak{h}^{*}$, and recall that $P_{\gamma}=U(\mathfrak{g}) \otimes_{U(\mathfrak{h})} \mathbb{C}_{\gamma}$. Then $P_{\gamma}$ is projective in $\mathscr{W}$, and thus $\eta P_{\gamma}$ is projective in $\mathcal{W}(\mu)$. Also, $\eta P_{\gamma}$ is finitely generated: $\eta P_{\gamma}$ is generated by $1 \otimes 1$ if $\gamma \leq \mu$ and $\eta P_{\gamma}=0$ otherwise. Thus, $\eta P_{\gamma} \in \mathbb{O}$. Therefore, we can construct a resolution $P_{\bullet} \rightarrow M$ of $M$ such that $P_{i}=\bigoplus_{j=1}^{n_{i}} \eta P_{\gamma_{j}^{i}} \in \mathbb{O}$ for some $\gamma_{j}^{i} \in \mathfrak{h}^{*}$, which is projective in $\mathscr{W}(\mu)$.

Recall that modules in $\mathcal{O}$ decompose according to blocks. Let $\tilde{P}_{i}$ be the component of $P_{i}$ contained in $\mathscr{C}$. (If $\mathscr{C}$ is a truncated block, the component of $P_{i}$ corresponding to the full block will be contained in the truncation $\mathscr{C}$ since $P_{i} \in \mathscr{W}(\mu)$ and $\mathscr{C}$ is truncated at $\mu$.) Then, $\tilde{P}_{\bullet} \rightarrow M$ is a projective resolution in $\mathscr{W}(\mu)$ and lies entirely in $\mathscr{C}$.

4.2. Cohomology and quotient categories. Throughout this section, let $\mathscr{C}$ be an infinite block for the Category $\mathbb{O}$ with a maximal element. Then $\mathscr{C}$ is a highest weight category which contains enough projective objects. Let $\Lambda$ be the corresponding weight poset indexing the simple objects in $\mathscr{C}$. For $\lambda \in \Lambda$ let $P(\lambda)$ be the projective cover of $L(\lambda)$. Set $P=\oplus_{\lambda \in \Lambda} P(\lambda)$. Then $P$ is a progenerator for $\mathscr{b}$, and $\mathscr{C}$ is Morita equivalent to $\operatorname{Mod}(B)$, where $B=\operatorname{End}_{\mathscr{C}}(P)^{\text {op }}$. 
We now apply results as described in [Cline et al. 1988, Theorem 3.5]. Let $\Omega$ be a finite coideal, that is, $\Omega=\Lambda-\{\gamma \in \Lambda \mid \gamma \preceq \delta\}$ for some fixed $\delta \in \Lambda$. Consider $P_{\Omega}=\oplus_{\lambda \in \Omega} P(\lambda)$ and set $A=\operatorname{End}_{\mathscr{C}}\left(P_{\Omega}\right)^{\mathrm{op}}$. Then there exists an idempotent $e \in B$, corresponding to the sum of identity maps in $\operatorname{End}_{\mathscr{C}}(P(\lambda))$ with $\lambda \in \Omega$ such that $e B e=A$. Also, observe that the quotient category $\mathscr{C}(\Omega)=\operatorname{Mod}(A)$ is a highest weight category.

For $\lambda \in \Omega$, set $M_{\Omega}(\lambda)=e M(\lambda), L_{\Omega}(\lambda)=e L(\lambda)$, and $P_{\Omega}(\lambda)=e P(\lambda)$. Note that $P_{\Omega}(\lambda)$ is the projective cover of $L_{\Omega}(\lambda)$. The following proposition compares extensions between Verma modules and simple modules in $\mathscr{C}$ and $\mathscr{C}(\Omega)$. We remark that this result appears as [Cline et al. 2004, Corollary 3.5] with more finiteness restrictions.

Proposition 7. Let $\lambda, v \in \Omega$. For all $i \geq 0$,

$$
\operatorname{Ext}_{\mathscr{C}}^{i}(M(\lambda), L(v)) \cong \operatorname{Ext}_{\mathscr{C}(\Omega)}^{i}\left(M_{\Omega}(\lambda), L_{\Omega}(v)\right) .
$$

Proof. Let $\lambda, v \in \Omega$. According to [Doty et al. 2004, Theorem 2.2], there exists a first quadrant spectral sequence,

$$
E_{2}^{i, j}=\operatorname{Ext}_{B}^{i}\left(\operatorname{Tor}_{j}^{A}\left(B e, M_{\Omega}(\lambda)\right), L(v)\right) \Rightarrow \operatorname{Ext}_{A}^{i+j}\left(M_{\Omega}(\lambda), L_{\Omega}(v)\right),
$$

and by their [Theorem 4.5], $\operatorname{Tor}_{0}^{A}\left(B e, M_{\Omega}(\lambda)\right)=M(\lambda)$. We need to show that $\operatorname{Tor}_{j}^{A}\left(B e, M_{\Omega}(\lambda)\right)=0$ for $j \geq 1$. Then the spectral sequence above collapses and yields

$$
\operatorname{Ext}_{B}^{i}(M(\lambda), L(v)) \cong \operatorname{Ext}_{A}^{i}\left(M_{\Omega}(\lambda), L_{\Omega}(v)\right) .
$$

for $i \geq 0$ and $\lambda, v \in \Omega$, as required.

First we consider the case when $j=1$. Since $\mathscr{C}(\Omega)$ is a highest weight category, we may again invoke [Doty et al. 2004, Theorem 4.5] which states that $\operatorname{Tor}_{0}^{A}\left(B e, M_{\Omega}(\lambda)\right)=M(\lambda)$ and $M(\lambda)$ belongs to $\mathscr{X}$; see their [Section 3.1] for a definition of $\mathscr{X}$. Therefore, $\operatorname{Tor}_{1}^{A}\left(B e, M_{\Omega}(\lambda)\right)=0$ by their [Proposition 3.1(A)].

To show that $\operatorname{Tor}_{j}^{A}\left(B e, M_{\Omega}(\lambda)\right)=0$ for $j \geq 2$, we use induction on the ordering on the weights in $\Omega$. If $\lambda$ is a maximal weight (relative to $\preceq$, the order introduced in (2)), then $M_{\Omega}(\lambda)$ is the projective cover of $L_{\Omega}(\lambda)$ and $\operatorname{Tor}_{j}^{A}\left(B e, M_{\Omega}(\lambda)\right)=0$ for $j \geq 1$. Now suppose that $\operatorname{Tor}_{j}^{A}\left(B e, M_{\Omega}(\mu)\right)=0$ for $j \geq 1$ for all $\mu \succ \lambda$ for $\mu \in \Omega$. Consider the short exact sequence

$$
0 \rightarrow N \rightarrow P_{\Omega}(\lambda) \rightarrow M_{\Omega}(\lambda) \rightarrow 0
$$

where $N$ has a filtration by modules $M_{\Omega}(\mu)$ with $\mu \succ \lambda$. This induces a long exact sequence

$$
\cdots \leftarrow \operatorname{Tor}_{j-1}^{A}(B e, N) \leftarrow \operatorname{Tor}_{j}^{A}\left(B e, M_{\Omega}(\lambda)\right) \leftarrow \operatorname{Tor}_{j}^{A}\left(B e, P_{\Omega}(\lambda)\right) \leftarrow \ldots
$$


For $j \geq 1$, $\operatorname{Tor}_{j}^{A}\left(B e, P_{\Omega}(\lambda)\right)=0$, and for $j \geq 2$, $\operatorname{Tor}_{j-1}^{A}(B e, N)=0$ by the induction hypothesis. Thus from the long exact sequence we can conclude for $j \geq 2$ that $\operatorname{Tor}_{j}^{A}\left(B e, M_{\Omega}(\lambda)\right)=0$.

Let $L(\lambda)$ and $L(v)$ be simple $B$-modules with $\lambda, v \in \Omega$. Then $e L(\lambda) \neq 0$ and $e L(v) \neq 0$. Let

$$
\cdots \rightarrow P_{2} \rightarrow P_{1} \rightarrow P_{0} \rightarrow L(\lambda) \rightarrow 0
$$

be the minimal projective resolution of $L(\lambda)$ in $\mathscr{C}$. Set $\Omega^{n+1}(L(\lambda))$ to be the kernel of the map $P_{n} \rightarrow P_{n-1}$. By convention, we let $\Omega^{0}(L(\lambda))=L(\lambda)$. Under a suitable condition on the minimal projective resolution, we can compare extensions between simple modules in $\mathscr{C}$ and $\mathscr{C}(\Omega)$. This comparison depends on bounding the composition factors in the projective resolution of $L(\lambda)$. The following proposition provides such a bound.

Proposition 8. Let $\lambda, v \in \Lambda$.

(i) If $\operatorname{Ext}_{\mathscr{C}}^{n}(\operatorname{rad} M(\lambda), L(v)) \neq 0$ then $l(\lambda)-l(v) \leq n-1$.

(ii) If $\operatorname{Ext}_{\mathscr{C}}^{n}(L(\lambda), L(v)) \neq 0$ then $l(\lambda)-l(v) \leq n$.

Proof. In this proof we assume that $\mathscr{C}$ is a thick block. In the case that $\mathscr{b}$ is a thin block or a finite block, the proposition follows from similar arguments.

(i) We prove this by induction on $n$. Let $n=0$. Since $\operatorname{rad} M(\lambda)=M\left(\lambda_{1}\right)+M\left(\lambda_{1}^{\prime}\right)$, with notation as in Theorem 1(iv), $\operatorname{Hom}_{\mathscr{C}}(\operatorname{rad} M(\lambda), L(v)) \neq 0$ if and only if $v=\lambda_{1}$ or $\lambda_{1}^{\prime}$, whence $l(\lambda)-l(v)=-1$.

Assume the result is true for $n-1$ and all pairs of weights in $\Lambda$. Recall from (4) that $\operatorname{rad} M(\lambda)=M\left(\lambda_{1}\right)+M\left(\lambda_{1}^{\prime}\right)$ and $\operatorname{rad}^{2} M(\lambda)=M\left(\lambda_{2}\right)+M\left(\lambda_{2}^{\prime}\right)=\operatorname{rad} M\left(\lambda_{1}\right)=$ $\operatorname{rad} M\left(\lambda_{1}^{\prime}\right)$. Thus we have a short exact sequence

$$
0 \rightarrow \operatorname{rad} M\left(\lambda_{1}\right)=\operatorname{rad} M\left(\lambda_{1}^{\prime}\right) \rightarrow M\left(\lambda_{1}\right) \oplus M\left(\lambda_{1}^{\prime}\right) \rightarrow \operatorname{rad} M(\lambda) \rightarrow 0,
$$

where the inclusion sends $x$ to $(x,-x)$ and the surjection sends $(x, y)$ to $x+y$. This induces a long exact sequence

$$
\begin{aligned}
\cdots \rightarrow \operatorname{Ext}_{\mathscr{C}}^{n-1}\left(\operatorname{rad} M\left(\lambda_{1}\right), L(v)\right) & \rightarrow \operatorname{Ext}_{\mathscr{C}}^{n}(\operatorname{rad} M(\lambda), L(v)) \\
& \rightarrow \operatorname{Ext}_{\mathscr{C}}^{n}\left(M\left(\lambda_{1}\right) \oplus M\left(\lambda_{1}^{\prime}\right), L(v)\right) \rightarrow \cdots .
\end{aligned}
$$

Suppose $l(\lambda)-l(v)>n-1$. Then $l\left(\lambda_{1}\right)-l(v)=l(\lambda)+1-l(v)>n$. This implies $\operatorname{Ext}_{\mathscr{C}}^{n}\left(M\left(\lambda_{1}\right), L(v)\right)=0$ by Theorem 6, and similarly for $\lambda_{1}^{\prime}$. Also, $l\left(\lambda_{1}\right)-$ $l(v)>(n-1)+1$, so $\operatorname{Ext}_{\mathscr{C}}^{n-1}\left(\operatorname{rad} M\left(\lambda_{1}\right), L(v)\right)=0$ by induction. This implies $\operatorname{Ext}_{\mathscr{C}}^{n}(\operatorname{rad} M(\lambda), L(\nu))=0$.

(ii) The proof is again by induction on $n$. The result is clear for $n=0$. Assume it is true for $n-1$. Consider the short exact sequence

$$
0 \rightarrow \operatorname{rad} M(\lambda) \rightarrow M(\lambda) \rightarrow L(\lambda) \rightarrow 0 .
$$


This induces a long exact sequence

$\cdots \rightarrow \operatorname{Ext}_{\mathscr{C}}^{n-1}(\operatorname{rad} M(\lambda), L(v)) \rightarrow \operatorname{Ext}_{\mathscr{C}}^{n}(L(\lambda), L(v)) \rightarrow \operatorname{Ext}_{\mathscr{C}}^{n}(M(\lambda), L(v)) \rightarrow \cdots$

Suppose $l(\lambda)-l(v)>n$. Then $\operatorname{Ext}_{\mathscr{C}}^{n-1}(\operatorname{rad} M(\lambda), L(v))=0$ by part (i), and $\operatorname{Ext}_{\mathscr{C}}^{n}(M(\lambda), L(\mu))=0$ by Theorem 6. This implies $\operatorname{Ext}_{\mathscr{C}}^{n}(L(\lambda), L(v))=0$.

Recall $P_{\bullet} \rightarrow L(\lambda)$ is a minimal projective resolution of $L(\lambda)$. For $\gamma \in \Lambda$, if $L(\gamma)$ is a composition factor of hd $P_{j}$, then $\operatorname{Ext}_{\mathscr{C}}^{j}(L(\lambda), L(\gamma)) \neq 0$. Therefore, Proposition 8 gives a bound on the composition factors which can appear in hd $P_{j}$. This is the condition needed to compare extensions between simple modules in $\mathscr{C}$ and $\mathscr{C}(\Omega)$.

Proposition 9. Let $\lambda, v \in \Omega$ and define $N=\min \{|l(\lambda)-l(\gamma)|: \gamma \in \Lambda-\Omega\}-1$. Then, for $j=0,1, \ldots, N$,

$$
\operatorname{Ext}_{\mathscr{C}}^{j}(L(\lambda), L(v)) \cong \operatorname{Ext}_{\mathscr{C}(\Omega)}^{j}\left(L_{\Omega}(\lambda), L_{\Omega}(v)\right)
$$

Proof. We first claim that $B e P_{j}=P_{j}$ for $j=0, \ldots, N$. Note that $B e P_{j}=P_{j}$ if and only if hd $P_{j}$ contains no composition factors that are killed by the idempotent $e$. Suppose that $L(\gamma) \subseteq$ hd $P_{j}$. Then $\operatorname{Ext}_{\mathscr{b}}^{j}(L(\gamma), L(\lambda)) \neq 0$. From the proof of Theorem 6 and using the duality on $\mathcal{W}(\mu)$, we have

$$
\begin{aligned}
\operatorname{Ext}_{\mathscr{C}}^{j}(L(\gamma), L(\lambda)) & \cong \operatorname{Ext}_{\mathscr{W}(\mu)}^{j}(L(\gamma), L(\lambda)) \\
& \cong \operatorname{Ext}_{\mathscr{W}(\mu)}^{j}(L(\lambda), L(\gamma)) \cong \operatorname{Ext}_{\mathscr{C}}^{j}(L(\lambda), L(\gamma)) .
\end{aligned}
$$

Then Proposition 8 implies that $|l(\lambda)-l(\gamma)| \leq j$. Therefore, for $j \leq N, \gamma \in \Omega$, and so $e L(\gamma) \neq 0$. Since hd $\Omega^{j}(L(\lambda)) \cong$ hd $P_{j}$, we have $B e \Omega^{j}(L(\lambda))=\Omega^{j}(L(\lambda))$ for $j=0,1,2, \ldots, N$.

Let $j=0,1, \ldots, N-1$. Since $B e \Omega^{j}(L(\lambda))=\Omega^{j}(L(\lambda))$ there exists a short exact sequence [Doty et al. 2004, Theorem 3.2],

$$
0 \rightarrow \Omega^{j+1}(L(\lambda)) / B e \Omega^{j+1}(L(\lambda)) \rightarrow \operatorname{Tor}_{0}^{A}\left(B e, e \Omega^{j}(L(\lambda))\right) \rightarrow \Omega^{j}(L(\lambda)) \rightarrow 0 .
$$

Note that we are using that $\Omega^{1}\left(\Omega^{j}(L(\lambda))\right) \cong \Omega^{j+1}(L(\lambda))$. Since $\Omega^{j+1}(L(\lambda))=$ $B e \Omega^{j+1}(L(\lambda))$, we have

$$
\operatorname{Tor}_{0}^{A}\left(B e, e \Omega^{j}(L(\lambda))\right) \cong \Omega^{j}(L(\lambda)) .
$$


Finally, we can apply [Doty et al. 2004, Theorem 2.4(B)(ii)] and a dimension shifting argument twice (see [Benson 1998, Corollary 2.5.4]) to see that

$$
\begin{aligned}
\operatorname{Ext}_{B}^{j+1}(L(\lambda), L(v)) & \cong \operatorname{Ext}_{B}^{1}\left(\Omega^{j}(L(\lambda)), L(v)\right) \\
& \cong \operatorname{Ext}_{B}^{1}\left(\operatorname{Tor}_{0}^{A}\left(B e, e \Omega^{j}(L(\lambda))\right), L(v)\right) \\
& \cong \operatorname{Ext}_{A}^{1}\left(e \Omega^{j}(L(\lambda)), e L(v)\right) \\
& \cong \operatorname{Ext}_{A}^{1}\left(\Omega^{j}(e L(\lambda)), e L(v)\right) \\
& \cong \operatorname{Ext}_{A}^{j+1}(e L(\lambda), e L(v)) \\
& \cong \operatorname{Ext}_{A}^{j+1}\left(L_{\Omega}(\lambda), L_{\Omega}(v)\right) .
\end{aligned}
$$

To justify the step between lines 3 and 4 in the identifications above, observe that the idempotent functor $e(-): \operatorname{Mod}(B) \rightarrow \operatorname{Mod}(A)$ is exact. Moreover, $B e P_{j}=P_{j}$ for $j=1,2, \ldots, N$ so we have an exact sequence of projective $A$-modules:

$$
e P_{N} \rightarrow e P_{N-1} \rightarrow \ldots e P_{1} \rightarrow e P_{0} \rightarrow e L(\lambda) \rightarrow 0 .
$$

This implies that $e \Omega^{j}(L(\lambda)) \cong \Omega^{j}(e L(\lambda)) \oplus Q_{j}$, where $Q_{j}$ is a projective $A$ module for $j=0,1, \ldots, N$.

4.3. Extensions between simple modules. Let $\mathscr{C}$ be a finite block, an infinite block with a maximal element, or a truncation of an infinite block with a minimal element. Let $\Lambda$ be the weight poset of $\mathscr{b}$, with length function $l: \Lambda \rightarrow \mathbb{Z}$.

Theorem 10. Let $\lambda, v \in \Lambda$. Then,

(a) if $\mathscr{C}$ is a thick block or a finite block,

$$
\operatorname{dim} \operatorname{Ext}_{\mathscr{C}}^{n}(L(\lambda), L(v))=\#\{\gamma \in \Lambda \mid \gamma \preceq \lambda, v ; 2 l(\gamma)-l(\lambda)-l(v)=n\} ;
$$

(b) if $\mathscr{C}$ is a thin block,

$$
\operatorname{dim} \operatorname{Ext}_{\mathscr{C}}^{n}(L(\lambda), L(\nu))=\#\{\gamma \in \Lambda \mid \gamma \preceq \lambda, v ; 2 l(\gamma)-l(\lambda)-l(\nu)=n\}
$$

if $n \leq 2$ and equals zero otherwise.

In particular, $\operatorname{Ext}_{\mathscr{C}}^{n}(L(\lambda), L(v)) \neq 0$ only when $n \equiv(l(\lambda)-l(v))(\bmod 2)$.

Proof. Suppose $\mathscr{C}$ is an infinite block with a maximal element. Let $\Omega$ be a finite coideal of $\Lambda$ containing $\lambda, v$. From Proposition 7, we know $\operatorname{Ext}_{\mathscr{C}}^{i}(M(\lambda), L(v))=$ $\operatorname{Ext}_{\mathscr{C}(\Omega)}^{i}\left(M_{\Omega}(\lambda), L_{\Omega}(v)\right)$. For a fixed $n \in \mathbb{Z}_{>0}$, we assume that $\Omega$ is sufficiently large so that $\gamma \in \Omega$ for all $\gamma \in \Lambda$ with $|l(\lambda)-l(\gamma)| \leq n$. Then Proposition 9 implies that $\operatorname{Ext}_{\mathscr{C}}^{n}(L(\lambda), L(v)) \cong \operatorname{Ext}_{\mathscr{C}(\Omega)}^{n}\left(L_{\Omega}(\lambda), L_{\Omega}(v)\right)$.

Thus, by replacing $\mathscr{C}$ by a quotient category $\mathscr{C}(\Omega)$ where appropriate, we may assume that $\mathscr{C}$ is a highest weight category with finite weight poset $\Lambda$. Because the objects of $\mathscr{C}$ have finite composition length, $\mathscr{C}$ is closed under the duality $D$ on $\mathscr{W}(\mu)$. Define $A(\gamma)=D M(\gamma)$. 
Now apply [Cline et al. 1993, 3.5]:

$$
\operatorname{dim} \operatorname{Ext}_{\mathscr{C}}^{n}(L(\lambda), L(v))=\sum_{\substack{\gamma \in \Lambda, i, j \in \mathbb{Z} \geq 0 \\ i+j=n}} \operatorname{dim}_{\operatorname{Ext}_{\mathscr{C}}^{i}}^{i}(L(\lambda), A(\gamma)) \operatorname{dim} \operatorname{Ext}_{\mathscr{C}}^{j}(M(\gamma), L(v)) .
$$

Using the duality on $\mathscr{C}, \operatorname{Ext}_{\mathscr{C}}^{i}(L(\lambda), A(\gamma)) \cong \operatorname{Ext}_{\mathscr{C}}^{i}(M(\gamma), L(\lambda))$. Then Theorem 6 gives the result.

4.4. $\mathbf{E x t}^{1}$-quivers. Let $\mathscr{b}$ be a finite block, a quotient of an infinite block with a maximal element, or a truncation of an infinite block with a minimal element. Let $\Lambda$ be the (finite) weight poset of $\mathscr{C}$. The $\operatorname{Ext}^{1}$-quiver of $\mathscr{C}$ is defined to be the directed graph with vertices labelled by $\Lambda$ and with $\operatorname{dim}_{\operatorname{Ext}_{\mathscr{C}}^{1}}^{1}(L(\lambda), L(\mu))$ edges from $\lambda$ to $\mu$. It is clear from Theorem 10 and Proposition 9 that the $\operatorname{Ext}^{1}$-quiver of $\mathscr{b}$ is obtained from the poset $\Lambda$ simply by replacing each edge by a pair of directed edges, one pointing in each direction.

The edges from $\lambda$ to $\mu$ can also be viewed as representing linearly independent elements of $\operatorname{Hom}_{\mathscr{C}}(P(\lambda), P(\mu))$ in the finite dimensional algebra

$$
A=\operatorname{End}_{\mathscr{C}}\left(\bigoplus_{\lambda \in \Lambda} P(\lambda)\right)^{\text {op }} \text {. }
$$

One can ask for the relations that exist between the maps in this algebra, which provides a presentation of the algebra by the quiver with relations.

Suppose that $\mathscr{b}$ is either a finite block or a finite quotient or truncation of a thin block. Then $\Lambda$ is a simple chain, say of length $n$, and it is quite easy to write down the structure of the projective indecomposables $P(\lambda)$. This is done for $n=4$ in [Futorny et al. 2001], and the pattern is the same for any $n$. Moreover, if the elements of $\Lambda$ are numbered $\lambda_{1}, \ldots, \lambda_{n}$ from top to bottom, and if $\alpha_{i}$ and $\beta_{i}$ represent the maps from $P\left(\lambda_{i}\right)$ to $P\left(\lambda_{i+1}\right)$ and from $P\left(\lambda_{i+1}\right)$ to $P\left(\lambda_{i}\right)$, respectively, for $1 \leq i \leq n-1$, then one sees easily that, up to scalar multiples,

$$
\alpha_{1} \beta_{1}=0 \quad \text { and } \quad \beta_{i} \alpha_{i}=\alpha_{i+1} \beta_{i+1} \quad \text { for } 1 \leq i \leq n-2
$$

Note that maps compose left-to-right, because of the () ${ }^{\text {op }}$ in the definition of $A$.

Now we can assume we're working in the basic algebra with simple modules (respectively, projective indecomposable modules) labelled by $\widehat{L}\left(\lambda_{i}\right)$ (respectively, $\left.\widehat{P}\left(\lambda_{i}\right)\right)$ for $i=1,2, \ldots, n$. Note that $\operatorname{dim} \widehat{L}\left(\lambda_{i}\right)=1$ for every $i$ so that $\operatorname{dim} A=$ $\sum_{i=1}^{n} \operatorname{dim} \widehat{P}\left(\lambda_{i}\right)$, which is easy to compute using the known structures of the $\widehat{P}\left(\lambda_{i}\right)$. On the other hand, using the relations given in (9), one can check directly that there are at most $\sum_{i=1}^{n} \operatorname{dim} \widehat{P}\left(\lambda_{i}\right)$ linearly independent words in the $\alpha_{i}$ and $\beta_{i}$. Thus (9) must be all the relations.

In contrast, suppose that $\mathscr{b}$ is a finite quotient or truncation of a thick block. Then the poset $\Lambda$ is isomorphic to the Bruhat order on a dihedral group. In this 
case the structure of the projectives, and the exact nature of the relations, seem to be quite difficult to deduce. For example, Stroppel [2003] works out the relations for the $\mathrm{Ext}^{1}$-quiver of the regular blocks of Category $\mathcal{O}$ for the finite simple complex rank 2 Lie algebras, using some deep results of Soergel. Not only are the answers quite complicated (for example, for $G_{2}$ there are 70 relations), but as far as we are aware the analogs of Soergel's results are not known for the Virasoro algebra. Nonetheless, based on Stroppel's computations, we speculate that the relations in the $\operatorname{Ext}^{1}$-quiver of $\mathscr{C}$ are all quadratic.

\section{Kazhdan-Lusztig theories and Koszulity}

5.1. Let $B=B_{0} \oplus B_{1} \oplus \cdots \oplus B_{q}$ be a finite-dimensional graded algebra, and let $\mathscr{C}_{B}^{g r}$ be the category of finite-dimensional graded $B$-modules. Regard every simple $B$-module $L$ as concentrated in degree zero; then the simple modules in $\mathscr{C}_{B}^{g r}$ can be obtained by shifting the gradings of the simple $B$-modules. If $L$ is a simple $B$-module then $L(i)$ will denote the simple module in $\mathscr{C}_{B}^{g r}$ by shifting $i$ places to the right; see [Cline et al. 1997, Section 1.3]. The algebra $B$ is Koszul if for all simple $B$-modules $L$ and $L^{\prime}$ and $m, n, p \in \mathbb{Z}$,

$$
\operatorname{Ext}_{\mathscr{C}_{B}^{g r}}^{p}\left(L(m), L^{\prime}(n)\right) \neq 0 \Rightarrow n-m=p .
$$

Now let $\mathscr{C}$ be either a finite block of $\mathcal{O}$, a truncation of an infinite block of $\mathscr{O}$ with a minimal element, or a quotient of an infinite block of $\mathbb{O}$ with a maximal element. Then $\mathscr{C}$ is a highest weight category (with duality) having a finite weight poset $\Lambda$ and length function $l$. Moreover, Theorem 6 implies that $\mathscr{C}$ has a Kazhdan-Luzstig theory, as defined in [Cline et al. 1993]. Recall that $\mathscr{C}$ is equivalent to $\operatorname{Mod}(A)$ for a finite-dimensional algebra $A$. Let gr $A$ be the associated graded algebra obtained by using the radical filtration on $A$. Moreover, set $L=\bigoplus_{\lambda \in \Lambda} L(\lambda)$, and define the homological dual of $A$ to be $A^{!}=\operatorname{Ext}_{\mathscr{C}}^{\bullet}(L, L)$. The following theorem establishes Koszulity results on $A$.

Theorem 11. Let $\mathscr{C}$ be as described above, and let $A$ be the associated quasihereditary algebra. Then

(a) $A^{!}$is Koszul,

(b) gr $A$ is Koszul, and

(c) $\left(A^{!}\right)^{!} \cong \operatorname{gr} A$.

Proof. To prove the theorem, it suffices to check the condition $\left(\mathrm{SKL}^{\prime}\right)$

$$
\operatorname{Ext}_{\mathscr{C}}^{n}\left(\operatorname{rad}^{i} M(\lambda), L(\mu)\right) \neq 0 \Rightarrow n \equiv l(\mu)-l(\lambda)+i(\bmod 2) \text { for all } \lambda, \mu \in \Lambda .
$$

In principle, that the same parity vanishes for $\operatorname{Ext}_{\mathscr{C}}^{n}\left(L(\lambda), A(\mu) / \operatorname{soc}^{i} A(\mu)\right)$ should also be checked, but this follows by duality in our setting. 
Once (SKL') is established, then by [Cline et al. 1997, Lemma 2.1.5] $\mathscr{C}_{A^{!}}^{g r}$ has a graded KL-theory, and so $\mathscr{C}_{A^{!}}^{g r}$ and $A^{!}$are Koszul using [Cline et al. 1994, Theorem 3.9]. The condition (SKL') implies parts (b) and (c) by [Cline et al. 1997, Theorem 2.2.1].

The case $i=0$ of (SKL') follows immediately from Theorem 6. Assume $i>0$. Then $\operatorname{rad}^{i} M(\lambda)$ is either 0 , a Verma module $M(v)$ with $l(v)-l(\lambda)=i$, or a sum of two such Verma modules. The first case is trivial, and in the second, we can use the same argument as for $i=0$. So assume we are in the third case. Let $\lambda_{i}$ and $\lambda_{i}^{\prime}$ be the two elements $v$ satisfying $l(v)-l(\lambda)=i$. We have a short exact sequence

$$
0 \rightarrow M\left(\lambda_{i}\right) \rightarrow \operatorname{rad}^{i} M(\lambda) \rightarrow L\left(\lambda_{i}^{\prime}\right) \rightarrow 0 .
$$

The corresponding long exact sequence is

$$
\begin{aligned}
\cdots \rightarrow \operatorname{Ext}_{\mathscr{C}}^{n}\left(L\left(\lambda_{i}^{\prime}\right), L(\mu)\right) & \rightarrow \operatorname{Ext}_{\mathscr{C}}^{n}\left(\operatorname{rad}^{i} M(\lambda), L(\mu)\right) \\
& \rightarrow \operatorname{Ext}_{\mathscr{C}}^{n}\left(M\left(\lambda_{i}\right), L(\mu)\right) \rightarrow \cdots,
\end{aligned}
$$

and we are assuming the middle term is nonzero. Then one of the two adjacent terms must be nonzero. If the term on the right is nonzero, then the same argument as for $i=0$ gives the desired parity condition, since $l\left(\lambda_{i}\right)=l(\lambda)+i$. If the term on the left is nonzero, then by Theorem 10 we have $n \equiv l\left(\lambda_{i}^{\prime}\right)-l(\mu) \equiv l(\lambda)+i-l(\mu)$ $(\bmod 2)$, which is equivalent to the required condition.

Remarks. 1. Since this proof holds for all quotient categories of $\mathscr{C}$, this shows that $\mathscr{C}$ satisfies the strong Kazhdan Lusztig condition; see [Cline et al. 1997, 2.4.1].

2. Suppose $\mathscr{b}$ comes from either a finite or a thin block of $\mathbb{O}$. Since the relations in the Ext ${ }^{1}$-quiver of $\mathscr{C}$ are all homogeneous (in fact quadratic; see (9)), it follows that $A$ itself is tightly graded (that is, $A \cong \operatorname{gr} A$ ). In particular in this case $A$ is Koszul.

3. An interesting open question is to determine whether $A$ is tightly graded or whether $A$ itself is Koszul when $A$ is associated to a thick block. The answers would be affirmative if the relations are all quadratic, as speculated in Section 4.4.

\section{References}

[Bey̆linson and Bernstein 1981] A. Běllinson and J. Bernstein, "Localisation de $g$-modules", C. R. Acad. Sci. Paris Sér. I Math. 292:1 (1981), 15-18. MR 82k:14015 Zbl 0476.14019

[Beilinson et al. 1996] A. Beilinson, V. Ginzburg, and W. Soergel, "Koszul duality patterns in representation theory", J. Amer. Math. Soc. 9:2 (1996), 473-527. MR 96k:17010 Zbl 0864.17006

[Benson 1998] D. J. Benson, Representations and cohomology. I, Second ed., Cambridge Studies in Advanced Mathematics 30, Cambridge University Press, Cambridge, 1998. MR 99f:20001a Zbl 0908.20001 
[Bernšteĭn et al. 1975] I. N. Bernšteĭn, I. M. Gel'fand, and S. I. Gel'fand, "Differential operators on the base affine space and a study of $\mathfrak{g}$-modules", pp. 21-64 in Lie groups and their representations (Budapest, 1971), edited by I. M. Gel'fand, Halsted, New York, 1975. MR 58 \#28285 Zbl 0338.58019

[Bernštel̆n et al. 1976] I. N. Bernšteĭn, I. M. Gel'fand, and S. I. Gel'fand, "A certain category of g-modules", Funct. Anal. Appl. 10 (1976), 87-92. Zbl 0353.18013

[Boe and Hunziker 2006] B. D. Boe and M. Hunziker, "Kostant modules in blocks of category O $S$ ", Preprint, 2006. arXiv math.RT/0604336

[Brylinski and Kashiwara 1981] J.-L. Brylinski and M. Kashiwara, "Kazhdan-Lusztig conjecture and holonomic systems”, Invent. Math. 64:3 (1981), 387-410. MR 83e:22020 Zbl 0473.22009

[Cline et al. 1988] E. Cline, B. Parshall, and L. Scott, "Finite-dimensional algebras and highest weight categories”, J. Reine Angew. Math. 391 (1988), 85-99. MR 90d:18005 Zbl 0657.18005

[Cline et al. 1993] E. Cline, B. Parshall, and L. Scott, "Abstract Kazhdan-Lusztig theories", Tohoku Math. J. (2) 45:4 (1993), 511-534. MR 94k:20079 Zbl 0801.20013

[Cline et al. 1994] E. Cline, B. Parshall, and L. Scott, "The homological dual of a highest weight category”, Proc. London Math. Soc. (3) 68:2 (1994), 294-316. MR 94m:20093 Zbl 0819.20045

[Cline et al. 1997] E. Cline, B. Parshall, and L. Scott, "Graded and non-graded Kazhdan-Lusztig theories", pp. 105-125 in Algebraic groups and Lie groups, edited by G. Lehrer et al., Austral. Math. Soc. Lect. Ser. 9, Cambridge Univ. Press, Cambridge, 1997. MR 99h:16015 Zbl 0880.16004

[Cline et al. 2004] E. Cline, B. Parshall, and L. Scott, "On Ext-transfer for algebraic groups", Transform. Groups 9:3 (2004), 213-236. MR 2005e:20069 Zbl 1063.20049

[Doty et al. 2004] S. R. Doty, K. Erdmann, and D. K. Nakano, "Extensions of modules over Schur algebras, symmetric groups and Hecke algebras", Algebr. Represent. Theory 7:1 (2004), 67-100. MR 2005e:20004 Zbl 1084.20004

[Fer̆gin and Fuchs 1988] B. L. Fer̆gin and D. B. Fuchs, "Cohomology of some nilpotent subalgebras of the Virasoro and Kac-Moody Lie algebras”, J. Geom. Phys. 5:2 (1988), 209-235. MR 91c:17012 Zbl 0692.17008

[Fer̆gin and Fuchs 1990] B. L. Fer̆gin and D. B. Fuchs, "Representations of the Virasoro algebra", pp. 465-554 in Representation of Lie groups and related topics, edited by A. M. Vershik and D. P. Zhelobenko, Adv. Stud. Contemp. Math. 7, Gordon and Breach, New York, 1990. MR 92f:17034 Zbl 0722.17020

[Futorny et al. 2001] V. Futorny, D. K. Nakano, and R. D. Pollack, "Representation type of the blocks of category O", Q. J. Math. 52:3 (2001), 285-305. MR 2002h:17006 Zbl 1021.17008

[Gončarova 1973a] L. V. Gončarova, "Cohomology of Lie algebras of formal vector fields on the line”, Funkcional. Anal. i Priložen. 7:2 (1973), 6-14. In Russian; translated in Funct. Anal. Appl. 7 (1973), 91-97. MR 49 \#4058a Zbl 0284.17006

[Gončarova 1973b] L. V. Gončarova, "Cohomology of Lie algebras of formal vector fields on the line”, Funkcional. Anal. i Priložen. 7:3 (1973), 33-44. MR 49 \#4058b Zbl 0289.57026

[Irving 1990] R. S. Irving, "BGG algebras and the BGG reciprocity principle", J. Algebra 135:2 (1990), 363-380. MR 92d:17011 Zbl 0725.17007

[Irving 1992] R. S. Irving, “Graded BGG algebras", pp. 181-200 in Abelian groups and noncommutative rings, Contemp. Math. 130, Amer. Math. Soc., Providence, RI, 1992. MR 94e:16013

[Kazhdan and Lusztig 1979] D. Kazhdan and G. Lusztig, "Representations of Coxeter groups and Hecke algebras", Invent. Math. 53:2 (1979), 165-184. MR 81j:20066 Zbl 0499.20035 
[Kumar 2002] S. Kumar, Kac-Moody groups, their flag varieties and representation theory, Progress in Mathematics 204, Birkhäuser, Boston, 2002. MR 2003k:22022 Zbl 1026.17030

[Lepowsky 2005] J. Lepowsky, "From the representation theory of vertex operator algebras to modular tensor categories in conformal field theory", Proc. Natl. Acad. Sci. USA 102:15 (2005), 53045305. MR 2006a:17027 Zbl 1112.17030

[Moody and Pianzola 1995] R. V. Moody and A. Pianzola, Lie algebras with triangular decompositions, Canadian Mathematical Society Series of Monographs and Advanced Texts, John Wiley \& Sons, New York, 1995. MR 96d:17025 Zbl 0874.17026

[Rocha-Caridi and Wallach 1983a] A. Rocha-Caridi and N. R. Wallach, "Characters of irreducible representations of the Lie algebra of vector fields on the circle", Invent. Math. 72:1 (1983), 57-75. MR 85a:17010 Zbl 0498.17010

[Rocha-Caridi and Wallach 1983b] A. Rocha-Caridi and N. R. Wallach, "Highest weight modules over graded Lie algebras: resolutions, filtrations and character formulas", Trans. Amer. Math. Soc. 277:1 (1983), 133-162. MR 84m:17005 Zbl 0512.17007

[Stroppel 2003] C. Stroppel, "Category 0: quivers and endomorphism rings of projectives", Represent. Theory 7 (2003), 322-345. MR 2004h:17007 Zbl 1050.17005

Received January 19, 2007. Revised July 23, 2007.

BRIAN D. BOE

DEPARTMENT OF MATHEMATICS

UNIVERSITY OF GEORGIA

ATHENS, GA 30602

brian@math.uga.edu

http://www.math.uga.edu/ brian/

DANIEL K. NAKANO

DEPARTMENT OF MATHEMATICS

UNIVERSITY OF GEORGIA

ATHENS, GA 30602

nakano@math.uga.edu

http://www.math.uga.edu/ nakano/

EMILIE WIESNER

Mathematics Department

Williams Hall 212, Ithaca College

953 DANBY ROAD

ITHACA, NY 14850

ewiesner@ithaca.edu

http://faculty.ithaca.edu/ewiesner/ 